\title{
DESENVOLVIMENTO E MEIO AMBIENTE: TECNOLOGIAS APROPRIADAS COMO O CAMINHO PARA A SUSTENTABILIDADE AMBIENTAL
}

\author{
Maurício A. Serra* \\ Gustavo Inácio de Moraes**
}

\section{INTRODUÇÃO}

O debate que leva ao amadurecimento definitivo do conceito de desenvolvimento sustentável tem suas raízes nos anos 60, especificamente em 1962, quando foi publicado o "Silent Spring"1 de Rachel Carson. Neste livro, Carson cita uma série de efeitos sobre o ambiente provocados pelo uso indiscriminado de produtos químicos na cadeia produtiva agrícola e industrial, sendo que, um dos efeitos mais danosos é a acumulação de resíduos tóxicos nos organismos vivos, expostos aos produtos que utilizam pesticidas. A autora constatou que várias espécies apresentavam crescentes frequiências de males cancerígenos, que produziam impactos diretos nas comunidades humanas na medida em que elas não só se alimentavam desses animais, como também dependiam da atividade pesqueira para sua sobrevivência econômica.

Embora este possa ser considerado o passo inicial, o conceito de sustentabilidade ganhou definitivamente um forte impulso após intenso debate dos anos 70 a respeito das relações entre crescimento econômico

* Doutor pela London School of Economics and Political Science. Professor do Programa de PósGraduação em Desenvolvimento Econômico (PPGDE) e do Departamento de Economia da Universidade Federal do Paraná. (serra@ufpr.br)

** Mestre em Desenvolvimento Econômico pelo PPGDE-UFPR e professor do Departamento de Economia da Universidade Federal do Paraná. (gustavoim@yahoo.com.br)

1 O texto de Rachel Carson (1907- 1964) inaugura, para muitos, o movimento ecologista. 
e meio ambiente, sendo que esta discussão foi estimulada pela publicação, em 1972, sob os auspícios do Clube de Roma, do livro "Os Limites do Crescimento", escrito pelo casal Dennis e Donella Meadows (1972). Estes autores confeccionaram cenários para cinco variáveis relevantes: dinâmica populacional, industrialização, recursos não-renováveis, consumo de calorias por pessoa e meio ambiente. A visão a respeito da manutenção do ritmo de crescimento econômico era pessimista, tendo como prognóstico o esgotamento do crescimento num prazo de aproximadamente um século caso ele fosse mantido naqueles moldes.

O colapso do sistema econômico seria acarretado principalmente pelos crescentes problemas ambientais e sociais. Os cenários elaborados tinham um tom alarmista, uma vez que, a expectativa propagada era a de que o mundo caminhava inevitavelmente para uma crescente e irreversível degradação ambiental, um aumento da má nutrição, uma dilapidação dos recursos naturais e um desmesurado crescimento populacional. A solução defendida para se evitar a catástrofe ambiental era a do congelamento do produto econômico e do crescimento da população, ou seja, o crescimento econômico zero e o estancamento da população eram as respostas corretas para um grave problema que se anunciava. É bem verdade, que ficou provado que o trabalho do casal Meadows tinha limitações sérias, o que tornava irreal a solução proposta. De fato, os argumentos defendidos no livro foram veementemente contestados pela corrente neoclássica do pensamento econômico, sobretudo por Solow (1974), que argumentava que a evolução tecnológica permitiria que o crescimento continuasse sem encontrar barreiras impeditivas. Além disso, ele igualmente refutava a possibilidade de crescimento zero, uma vez que, este resultaria na estagnação de condições presentes, o que acabaria por privilegiar determinados países e, conseqüentemente, manteria, sem qualquer alteração, a estrutura da economia mundial.

Apesar das limitações, é inegável a importância do trabalho do casal Meadows na medida em que ele pavimentou o caminho para a aceitação do conceito de desenvolvimento sustentável. A mais famosa definição de sustentabilidade é a da Comissão Mundial sobre Meio Ambiente e Desenvolvimento, que define desenvolvimento sustentável 
como sendo "aquele que atende às necessidades do presente sem comprometer a possibilidade de as gerações futuras atenderem as suas próprias necessidades" (CMMAD, 1988, p. 46). Na realidade, a noção de sustentabilidade surgiu como uma proposta conciliadora, uma vez em que há o reconhecimento de que o progresso tecnológico flexibiliza os limites ambientais, embora não os elimine, e que o crescimento econômico é uma condição importante e necessária, mas não suficiente para a completa eliminação da pobreza e das desigualdades sociais.

Nobre e Amazonas (2002) sublinham que por ser extremamente amplo e vago, a noção de sustentabilidade foi conveniente para agregar apoio de governos, das agências internacionais e da sociedade civil à idéia de que havia compatibilidade entre desenvolvimento e meio ambiente, o que acabou por oferecer um tremendo alívio ao intenso debate sobre os limites do crescimento dos anos 70. A ampla difusão e aceitação da noção de sustentabilidade mostra claramente que houve um certo avanço no que tange a importância da questão ambiental. Por outro lado, sua imprecisão conceitual implica em diferentes interpretações e da mesma forma na dificuldade concreta de se traduzir essa noção em termos de políticas públicas.

A questão fundamental continua sendo a capacidade de superação indefinida dos limites ambientais globais, ou seja, se o progresso científico e tecnológico é capaz de superar indefinidamente os obstáculos impostos pela disponibilidade de recursos naturais de modo a propiciar uma contínua expansão econômica dos países. Esta questão se torna ainda mais complexa em termos de viabilização da sustentabilidade quando se leva em consideração a existência de um padrão norte-sul de consumo e de um gap tecnológico entre os países.

O objetivo deste artigo é apresentar os pontos de vista a respeito do alcance da sustentabilidade vis-à-vis o crescimento e a própria evolução da produção econômica e qual o papel que a tecnologia pode assumir para contribuir com a sustentabilidade.

Para atingir tal objetivo, o artigo foi dividido em três seções. Na primeira seção discute-se o comportamento da degradação ambiental em função do crescimento econômico, destacando a hipótese estabelecida 
através da formulação da Curva Ambiental de Kuznets. Na segunda seção serão comparados os níveis de consumo ora em voga nos países desenvolvidos e nos países em desenvolvimento e seus impactos sobre o desenvolvimento sustentável. Finalmente, a terceira seção destacará como a tecnologia poderá auxiliar a busca por um desenvolvimento sustentável em um padrão de crescimento econômico contínuo e com uma permanente pesquisa por condições de consumo melhores por parte dos países em desenvolvimento.

\section{DESENVOLVIMENTO VERSUS DEGRADAÇÃO AMBIENTAL: A CURVA AMBIENTAL DE KUZNETS}

A formulação da Curva Ambiental de Kuznets foi inspirada na original Curva de Kuznets, proposta em 1954 por Simon Kuznets, pela qual obteve grande reconhecimento, sendo inclusive premiado com o Nobel de Economia. Kuznets (1955) discutia o perfil da distribuição de renda durante os diferentes estágios de uma economia, observando o processo histórico da construção do capitalismo, aceitando a idéia de que as economias transitam de um estágio em que são predominantemente agrícolas para outro estágio onde se tornam economias industriais e pujantes. Isso foi especialmente verdadeiro para os países que Kuznets observou: Alemanha, Inglaterra e Estados Unidos, que em diferentes épocas viveram o processo de transição de economia agrícola para economia industrial. Naturalmente, a riqueza criada neste processo não foi distribuída de forma igualitária, mas Kuznets notou algo curioso nos três países: a distribuição de renda possuía um comportamento não-linear, descrevendo uma parábola com concavidade voltada para baixo, ou em forma de $\mathrm{U}$ invertido, ao longo do processo de transição de economia agrícola, de baixa renda per capita, para economia industrializada, com alta renda per capita. Na seqüência do seu estudo, uma série de verificações empíricas foram feitas e, enquanto umas aceitavam a hipótese da evolução em forma de $\mathrm{U}$ invertido,a grande maioria rejeitou a hipótese, todavia sem encerrar o debate.

De forma análoga à proposição de Kuznets(1955), Grossman e Krueger (1991), World Bank (1992) e Shafik e Bandyopahyay (1992) propõe a Curva Ambiental de Kuznets, conhecida pelas iniciais em inglês 
EKC, onde ao invés de desigualdade de renda, eram tomadas como variáveis dependentes a degradação ambiental medida por poluição ambiental e de bacias hidrográficas, e permanecendo a renda per capita como variável independente. Desse modo foi estabelecida uma relação não-linear característica (U invertido, ou parábola com concavidade voltada para baixo) que apontava que em determinado nível de renda per capita os indicadores de degradação ambiental se tornariam declinantes.

A razão deste comportamento seria justificada de diversas maneiras : num processo de desenvolvimento econômico seria inevitável que agressões ao meio ambiente ocorressem; contudo, à medida que a renda continuasse se elevando as pessoas adquiririam maior consciência sobre os danos ao seu próprio ecossistema do processo de desenvolvimento econômico exigindo, por conseqüência, regulamentos mais firmes, bem como premiando 0 consumo de produtos que fossem ambientalmente inofensivos ou que fossem produzidos através de processos que levassem em conta as externalidades sobre o ecossistema. Portanto, a proteção ao ambiente poderia ser tomada como um bem normal, aquele cujo consumo aumenta na medida em que a renda se eleva. Vale lembrar que a valoração dos recursos naturais traria Uma segunda explicação não excludente em relação à primeira, é que as empresas adotariam tecnologias que privilegiassem processos limpos, em função de custo e da produtividade apresentadas. Este é o caso, sobretudo, para novas fontes energéticas e biotecnologia, na área farmacêutica e de cosméticos. Uma terceira explicação, também não excludente em relação à primeira, residiria no fato de que ao ocorrer elevação de renda o perfil aquisitivo da região se modificaria, preferindo importar os bens cuja produção cause impactos ao ambiente, ao invés de produzi-los em seu território. As explicações são basicamente levantadas por Panayotou (1997) e Bousquet e Favard (2000). Suri e Chapman (1998) estudam relações nesse sentido para a produção de produtos cuja linha de processo é energo-intensiva. Os autores levam em conta como variável explicativa a intensidade energética per capita e a participação das exportações e importações em relação ao nível de produção para dois grupos de países, divididos em alta renda e baixa/média renda. A curva ambiental de Kuznets é verificada, porém regra geral mostra que a renda per capita necessária ao ponto de decrescimento 
é elevada, situando-se entre US\$ 55,5 mil e US\$ 224,16 mil, níveis estes tomados considerando o poder de paridade de compra.

Tem sido entretanto explorada a idéia de que não apenas a renda per capita determinaria o nível de degradação ambiental, mas também outros fatores tais como a desigualdade de renda e o perfil tecnológico da estrutura produtiva. As investigações quanto ao efeito da desigualdade foram principalmente estabelecidas por Torras e Boyce (1998) e Bousquet e Favard (2000) que incluíram a desigualdade de renda como variável explicativa da degradação ambiental. Ambos porém não conseguem estabelecer de forma clara um comportamento padrão. Vale lembrar contudo, que inserir a desigualdade de renda como variável explicativa pode tornar a especificação incorreta, uma vez que a curva original de Kuznets relacionava desigualdade de renda ao nível de renda per capita e presumindo que a relação não-linear entre essas variáveis seja válida, haveria expressiva chance de caracterizarmos uma multicolinearidade entre essas variáveis. De qualquer forma, é de se considerar que a formulação original, tanto para desigualdade de renda, como para degradação ambiental, diz respeito somente ao nível de renda coeteris paribus ${ }^{2}$.

Uma limitação que se coloca em relação à Curva Ambiental de Kuznets é que em geral os estudos tomam como critério a ser adotado variáveis que têm caráter cumulativo e não apenas passageiro. É exemplar, desse caráter, o estudo de Shafik e Bandyopadhyay (1992), que entre alguns critérios adota o desmatamento como variável a ser explicada e que portanto teria que apresentar o formato de $U$ invertido, ou de relação não linear característica. Todavia os autores desconsideraram o fato de que, regra geral, o desmatamento tem sido irreversível ${ }^{3}$, já que as fronteiras agrícolas e urbanas tem se expandido de forma contínua ${ }^{4}$. O mesmo

2 Embora a desigualdade tenha impactos sobre o consumo e a intensidade energética. Um estudo interessante sobre o tema é "Energia e Classes Sociais no Brasil”, de Antônio Carlos Boa Nova, ed. Loyola, 1985.

3 Cabe considerar que reflorestamento é possível. No entanto, exige longa maturação, vide o caso da indústria de celulose, e certamente não seria feito na área original, por exemplo a área quando do início da colonização européia na América.

4 Esse foi o caso ao longo da história nos Estados Unidos e Europa, principalmente, onde a cobertura vegetal original é ínfima. 
raciocínio valeria para um estudo que tomasse como variável explicativa a extinção de espécies vegetais e animais ${ }^{5}$, que são perdas irreversíveis do ponto de vista de processos naturais ${ }^{6}$. Assim não é surpresa que Shafik e Bandyopadhyay (1992) não obtivessem a curva de Kuznets para o critério desmatamento. Por isso, é válido alertar para um exame mais atento a respeito do critério utilizado, detalhando-o e classificando-o inicialmente como de efeitos permanentes ou de efeitos passageiros. Nesses últimos, os que realmente contariam para a relação proposta pela Curva Ambiental de Kuznets, poderíamos enquadrar poluição das águas e do ar ou até mesmo ocorrência de doenças respiratórias nestes critérios de efeitos passageiros.

Em contraposição, Grossman e Krueger (1991) foram aqueles que obtiveram resultados mais robustos na investigação da Curva Ambiental de Kuznets. Os resultados dessa pesquisa validaram a Curva Ambiental de Kuznets para os indicadores de $\mathrm{SO}_{2}$ nas partículas de ar, para fumaça, oxigênio dissolvido na água, demanda biológica por oxigênio na água e partículas de coliformes fecais na água; todavia, para partículas suspensas no ar o resultado foi uma curva negativamente relacionada à renda per capita. Nos cinco critérios onde a Curva Ambiental de Kuznets foi verificada o ponto de máximo, ou seja onde houve a inversão de relação positiva para relação negativa esteve situado entre US\$2,7 mil e US\$ 8 mil, indicando a possibilidade de países ainda em estágio intermediário de desenvolvimento alcançarem trajetória de declínio na degradação ambiental.

Por fim, citaremos Moomaw e Unruh (1997) que realizam trabalho tomando como critério a presença de gás carbônico $\left(\mathrm{CO}_{2}\right)$, ou, no ar e procuram pela Curva Ambiental de Kuznets, dividindo os países em amostras particulares e testando três tipos de especificações: de efeitos fixos, polinomial quadrática e polinomial cúbica. Os autores recorrem a uma técnica de painel e dividem os países em grupos denominados tipo 1, que seria composto por países membros da OCDE, países tipo 2, grupo formado por economias

Por exemplo, anfíbios, a espécie mais exposta a mudanças climáticas.

6 Os defensores da tecnologia como meio de mitigar efeitos danosos sobre o ambiente poderiam argumentar que a clonagem é uma alternativa. Por isso pedimos atenção ao termo "do ponto de vista de processos naturais". 
em desenvolvimento, e países do tipo 3, predominantemente pobres. Para a especificações de efeitos fixos e polinomial quadrática, Moomaw e Unruh (1997) encontram a Curva Ambiental de Kuznets apenas para os países do grupo 1, enquanto para países do grupo 2 e 3 a relação não foi verificada. Acrescente-se ainda que na forma polinomial cúbica, a curva Ambiental de Kuznets não surgiu sequer para os países do grupo 1, sugerindo que a adoção desta especificação invalide a relação. À luz dos resultados obtidos, vale destacar duas limitações do resultado : a primeira destacada pelos autores mostra que a renda não parece ser o fator determinante para a determinação de um ponto de máximo na presença de $\mathrm{CO}_{2}$ na atmosfera, pois todos os países do grupo 1 observaram a mudança de relação positiva para negativa simultaneamente, derrubando aparentemente a condição de coeteris paribus; a segunda limitação ${ }^{7}$ é que o fator determinante para a existência da relação de $U$ invertido é a estrutura da economia e não necessariamente o nível de renda per capita, afinal os países do grupo 1 foram os únicos a completar a transição de economias agrícolas para uma economias de serviços, passando intermediariamente pelo processo de industrialização. Desse modo a renda per capita funcionaria como uma proxy desses processos, tal como proposto na formulação original de Kuznets $(1955)^{8}$, limitando a amostra apenas aos países que completaram a transição estrutural de suas economias.

Cabe notar que desde a proposição da Curva Ambiental de Kuznets em 1991, os estudos empíricos realizados na maioria das vezes acabaram refutando a possibilidade da existência da relação entre degradação ambiental e renda per capita em forma de parábola com concavidade voltada para baixo, ou U invertido. Alguns estudos, porém, identificam outros fatores causadores de inversão que não a renda per capita, alguns deles ainda relacionados ao crescimento econômico, como tecnologia, outros não necessariamente ligados ao crescimento econômico. De qualquer forma, o

7 E passível de analogia com os estudos que procuram a curva original de Kuznets, que relacionam desigualdade e renda per capita.

8 Portanto, a passagem de renda per capita de US\$ 120 para US\$ 600 pouca coisa significaria em termos de estrutura econômica, permanecendo predominantemente agrícola, embora a renda fosse quintuplicada. 
debate sobre degradação ambiental, reconheça-se, foi enriquecido pelas proposições de Grossman e Krueger (1991) e World Bank (1992) e estabelece um novo referencial para pesquisas empíricas sobre o tema.

\section{PADRÕES DE CONSUMO NORTE E SUL: O HIATO PODERÁ SER VENCIDO?}

Desde então, políticas públicas tem sido adotadas pelos governos dos diversos países na busca do desenvolvimento sustentável. Contudo, a forte diferenciação nos padrões de consumo permanece até os dias atuais. Por exemplo, examinando-se o consumo per capita de energia, em toneladas equivalentes de petróleo, nota-se uma diferença acentuada entre as nações mais ricas e as nações mais pobres (tabela1) .

\section{Tabela 1 - Consumo de Energia per Capita}

(Toneladas Equivalentes de Petróleo)

\begin{tabular}{|l|l|l|l|}
\hline Grupo de Países & 2000 & 2001 & 2002 \\
OCDE & 5,46 & 5,41 & 5,42 \\
América Latina e Caribe & 1,17 & 1,16 & 1,15 \\
Baixa e Média Renda & 0,96 & 0,96 & 0,99 \\
\hline
\end{tabular}

Fonte: World Bank, 2005.

Ao mesmo tempo, no ano 2000, as toneladas de $\mathrm{CO}_{2}$ produzidas pelos países da OCDE encontravam-se na casa de 12,45 toneladas per capita anuais, enquanto os países da América Latina lançavam na atmosfera “apenas" 2,66 toneladas per capita de $\mathrm{CO}_{2}$, e os países de baixa e média renda em seu conjunto lançavam 2,45 toneladas per capita de $\mathrm{CO}_{2}$ na atmosfera (World Bank, 2005). Ao mesmo tempo, a população dos países da OCDE representa aproximadamente apenas um sexto da população mundial, 919 milhões de pessoas, e com uma taxa de fertilidade inferior aos países de baixa e média renda (World Bank, 2005). O quadro agravase considerando que significativa parcela da população dos países em desenvolvimento permanecem localizadas no setor rural, onde a subsistência e 0 acesso a poucos bens de consumo são mais característicos.

Nesse ritmo, criam-se importantes dificuldades para se reproduzir o mesmo padrão de desenvolvimento nos países de baixa e média renda. 
Novas soluções técnicas terão obrigatoriamente que ser adotadas e/ou o padrão de consumo da sociedade deverá resultar em elementos diferenciados de consumo dos recursos. Desse modo, qualquer discussão que envolva um maior acesso aos bens necessários e/ou de luxo por parte das populações dos países em desenvolvimento passa por soluções tecnológicas. A dificuldade com este argumento é que grande parte dos países em desenvolvimento necessitam de recursos para desenvolver e aplicar na produção tais tecnologias. Ou quando as tecnologias já existem, o conhecimento e a patente é propriedade de um país desenvolvido, acarretando maiores dificuldades com o balanço de pagamentos. Um exemplo deste fenômeno é encontrado no indicador de investimento direto estrangeiro. No ano de 2003, os países de renda baixa e média receberam US\$ 165 bilhões, ao passo que os países desenvolvidos receberam mais que o dobro: US\$ 390 bilhões (WORLD BANK, 2005). Portanto, descapitalizados, os países em desenvolvimento se encontram numa situação ainda mais complicada para conseguir almejar um novo patamar tecnológico, qualquer que seja ele, superior em poupança de recursos ou superior na sua própria produção.

Outros indicadores que demonstram o corte existente entre países desenvolvidos e em desenvolvimento podem ser ilustrados por outros critérios. Em todos eles, evidencia-se o diferencial de consumo e o potencial impacto que haveria sobre o planeta no caso de estas populações acessarem o mesmo nível de consumo das populações dos países desenvolvidos.

A exemplificação de como os padrões de consumo podem contribuir para dificultar o alcance da sustentabilidade pode ser ilustrado mesmo dentro de uma sociedade. Dentro do caso brasileiro, encontramos alguns exemplos que ilustram a situação. Motta et alli (2001) ilustram como a possa de bens variou entre 1987 e 1996, pelos dados da Pesquisa de Orçamento Familiares. Na posse de automóveis, por exemplo, houve um crescimento de $112 \%$ na aquisição de automóveis nas famílias que percebiam entre 2 e 3 salários mínimos de renda, ao passo que nas classes com 30 ou mais salários houve um aumento de apenas $21 \%$. O mesmo padrão é verificado para a posse de refrigerador (57\% e 21\%, respectivamente) e para televisores (288\% e 24\%, respectivamente). Parte desse efeito, certamente, pode ser atribuído à adoção do Plano Real. Uma 
vez que estabilizou o nível de preços, o Real proporcionou que a camada da população que possuía menor renda pudesse, nesta nova situação, ter o seu padrão de aquisição protegido.

$\mathrm{Na}$ tabela 2, a seguir, demonstra-se a preocupação que a trajetória de crescimento econômico causa no que diz respeito à oferta de água. Ressaltese que a tabela considera as populações urbanas de cada segmento de países, sendo portanto sensível ao aumento da população urbana nos países de baixa e média renda, sobretudo por conseqüência da migração rural urbana. A migração rural urbana tende a se intensificar na medida em que a modernização agrícola (tabela 3) persiste nos países em desenvolvimento.

Tabela 2 - Acesso ao Serviço de Água Potável

\begin{tabular}{|l|c|c|}
\hline Grupo de Países & 1990 & 2002 \\
OCDE & 100 & 100 \\
América Latina e Caribe & 93 & 96 \\
Baixa e Média Renda & 93 & 93 \\
Baixa Renda & 86 & 89 \\
\hline
\end{tabular}

Fonte: World Bank, 2005.

Tabela 3 - Produtividade do Setor Agrícola (Valor Adicionado na Agricultura por Trabalhador), dólares de 2000

\begin{tabular}{|l|c|c|c|}
\hline Grupo de Países & $1989-91(\mathrm{~A})$ & $2001-03(\mathrm{~B})$ & $\begin{array}{c}\text { \% variação } \\
\text { (A/B) }\end{array}$ \\
OCDE & 11395 & 20544 & $80,2 \%$ \\
América Latina e Caribe & 1791 & 1869 & $4,3 \%$ \\
Baixa e Média Renda & 461 & 565 & $22,5 \%$ \\
Baixa Renda & 329 & 375 & $14,0 \%$ \\
\hline
\end{tabular}

Fonte: World Bank, 2005.

Para além desta relação, é importante ressaltar que expressiva parte do uso da água está centrada na agricultura, enquanto setor de atividade econômica. No mundo como um todo, o uso da água é 70\% localizado na agricultura, ao passo que nos países de baixa renda este índice chega a $92 \%$, distantes dos $42 \%$ das nações mais ricas. Já no setor industrial ocorre o inverso. Enquanto os países de baixa renda consomem apenas 
3\% da água potável na indústria, os países de alta renda consomem 42\% da água potável nos segmentos industriais. Estes números demonstram a necessidade de no desenvolvimento industrial dos países com baixa renda estarem contempladas.

\section{TECNOLOGIA E SUSTENTABILIDADE AMBIENTAL}

O objetivo desta seção é demonstrar duas leituras a respeito da evolução tecnológica que direcionam a produção técnica no sentido de se pouparem recursos naturais. Uma delas, divide-se em duas vertentes: primeiro, as inovações institucionais no sentido de estimularem novas tecnologias com orientação para a sustentabilidade e segundo, o desenvolvimento dos setores de tecnologia de comunicações e microeletrônica colocados como novos paradigmas. Estes seriam processos predominantes nos países centrais, ao passo que a adoção de uma tecnologia intermédia seria predominante nos países em desenvolvimento.

\section{Países Centrais}

Boa parte das análises do desempenho das tecnologias ainda se concentram no acompanhamento nas diferentes etapas de adoção de um novo processo produtivo ou um novo produto. A avaliação concentrariase em grau de alteração, grau de melhoramento, grau de diferenciação, grau de transformação e ainda grau de novidade (RIBAULT, MARINET e LEBIDOIS, 1995). Com a preocupação sustentabilidade ambiental a avaliação se daria na condição de melhoramento introduzido pela tecnologia.

Neste sentido, os novos paradigmas superam os antigos paradigmas produtivos, estabelecidos a partir da primeira revolução industrial e posteriormente a partir da adoção do fordismo/taylorismo. Ambos os paradigmas pressupunham produção em massa e consumo de matérias primas em larga escala, modificando-se apenas o patamar de intensidade entre um e outro. Por trás da intensificação do padrão fordista/taylorista encontravam-se importantes transformações tecnológicas, como a difusão da eletricidade e do motor à combustão. 
No final do século XX com um novo paradigma de firma surgindo, as condições de produção diferem-se. O processo organizacional das firmas concentra-se na configuração em rede e a tecnologia neste momento assume um caráter de causa e também de conseqüência nas transformações. Neste novo cenário a firma tem a sua disposição um "leque" de opções tecnológicas mais numerosas (Tigre, 1998). Portanto, a configuração em "rede" da firma passa a ser um fator decisivo. É partindo desta configuração que se possibilita à firma maximizar as fontes de informação e ter novas gestões sobre a tecnologia e sobre os fatores de produção, como a mão-de-obra. Por sua vez, a "rede" estabelecida pela firma assume diversos tipos de configuração ${ }^{9}$.

Neste sentido, as mudanças tecnológicas tendem a ter repercussões maiores e mais duradouras sobre o crescimento econômico. A primeira parte da importância das indústrias que ofertam insumos para vários segmentos seria o fato de que, desse modo, atuam reduzindo custos e aumentando a oferta.

Em paralelo, com a transformação produtiva concentrando-se em setores mais eficientes e com repercussões diferentes sobre o processo produtivo a sustentabilidade ambiental tende a ser favorecida. A busca por uma maior maximização no uso dos recursos tende a melhorar 0 aproveitamento dos recursos naturais e o maior peso dos setores micro eletrônicos e de comunicações tende a diminuir a intensidade no uso dos recursos naturais.

A análise de Jorgensen Christensen e Lau (1973), por exemplo, faz uma releitura da função de produção tradicional. A função de produção tradicional destaca o papel do capital e do trabalho na obtenção do produto. A nova função de produção proposta incorpora a necessidade de energia e materiais no processo produtivo. Assim sendo, reconhece-se, ao menos teoricamente, a participação e a importância dos recursos, em grande parte naturais, na obtenção do produto econômico.

A segunda parte, talvez mais relevante é que este processo associase a fatores organizacionais, socioculturais e institucionais. A análise de

9 As que mais se destacam seriam: alianças estratégicas, redes hierarquizadas e redes nãohierarquizadas. 
Dosi (1988), por exemplo, destaca o papel das macroinstituições em contraponto às microinstituições. As macro instituições operariam no nível das normas e regulamentações e até mesmo dentro da política econômica. As micro instituições por seu turno estariam ligadas ao processo produtivo no interior e na rotina da produção.

Nesta linha de raciocínio, Foxson et al. (2004), destacam a importância da adoção, por parte das instituições públicas, de incentivos que beneficiem o desenvolvimento de políticas de geração de tecnologia sustentável dentro dos SNI (Sistemas Nacionais de Inovação). Aqui, efetivamente destacamse os papéis da pesquisa, desenvolvimento e demonstração (PD \& D), e das demais fases da vida da tecnologia: desenvolvimento experimental, indústria crescente e indústria madura. Os autores concluem que o papel das políticas públicas de inovação são decisivos e devem ser orientados a longo prazo.

\section{Países Periféricos}

Nos países em desenvolvimento a adoção das novas configurações da firma estão encontrando limitações no que diz respeito a sua difusão. O primeiro elemento a condicionar este comportamento é o fato de que as indústrias que lideram este processo, microeletrônica e comunicações, encontrarem-se ainda em um estágio mais atrasado nos países em desenvolvimento. De outro lado, há que se considerar o fraco desempenho das instituições neste grupo de países e também o mercado consumidor pequeno como um fator de limitação, obrigando as indústrias com novos paradigmas tecnológicos a competir, em condições desfavoráveis, no mercado internacional.

Os países em desenvolvimento ausentes e retardatários nestas mudanças de caráter tecnológico, poderiam trilhar um caminho autônomo e respeitando suas limitações. Este caminho seria o da tecnologia intermédia, apontado em Schumacher (1979). A concepção do conceito de tecnologia intermédia envolveu uma preocupação com as populações que não eram participantes das vantagens do desenvolvimento econômico, através de seus instrumentos mais modernos, mas que por outro lado ao ingressarem 
no consumo buscassem um padrão de consumo mais correto em termos de aproveitamento dos recursos.

Torna-se importante observar a profunda relação entre a proposta de tecnologia intermédia e o caráter dualístico das economias em desenvolvimento, na quais convivem os setores moderno e o tradicional, que foi concebido por Lewis ${ }^{10}$ (1954). Portanto, se adotado o nível correto de tecnologia não haveria confronto entre recursos escassos e desenvolvimento econômico.

Uma importante abordagem da adoção da tecnologia intermédia é o direcionamento para as indústrias mais propensas a desenvolvimento. Todavia, a escolha estaria submetida a dois fatores de risco e de grande interesse para os países em desenvolvimento: a) que fosse poupadora de recursos econômicos; e b) que ao mesmo tempo fosse poupadora de recursos naturais. A implementação poderia ser realizada em diferentes regiões, respeitando os limites impostos pelas potencialidades locais.

Uma maneira mais viável, ao alcance dos países em desenvolvimento, seria não apenas reconhecer as limitações do salto brutal que se constitui a transição de um padrão de consumo mais baixo para um padrão de consumo mais alto, mas também preparar essa população excluída para ingressar de forma adequada no processo de desenvolvimento econômico e de consumo, sem que haja um aumento explosivo da dilapidação de recursos naturais.

\section{CONSIDERAÇÕES FINAIS}

Percebendo-se a necessidade do crescimento econômico e da extensão deste para os países em desenvolvimento, o impacto sobre o meio ambiente poderá se ampliar intensamente. A hipótese estabelecida pela Curva Ambiental de Kuznets, de que o nível crescente de renda per capita acaba por determinar uma diminuição no ritmo da degradação parece estar mais associada à incorporação de padrões tecnológicos do que à dinâmica do crescimento econômico.

${ }^{10}$ Curiosamente, Lewis (1984, p.130), no artigo em que faz uma revisão da sua obra, sublinha que os países em desenvolvimento necessitam adotar tecnologias apropriadas, mas ressalta que não comunga da mesma posição de Schumacher, que , segundo Lewis, tem uma "posição extremista". 
Os padrões de consumo dos países desenvolvidos quando reproduzidos para os países em desenvolvimento, tornam impossível a manutenção do ecossitema global em qualquer equilíbrio. Contudo, não se pode negar às populações dos países em desenvolvimento a oportunidade de acessar os bens de consumo e o conforto que as populações dos países desenvolvidos possuem.

A tecnologia seria, portanto, a principal resposta para o alcance da sustentabilidade, na medida em que proporcionasse que o consumo de bens e a produção econômica fosse orientado para padrões poupadores de recursos naturais. Todavia, mesmo estas tecnologias encontram-se predominantemente desenvolvidas nos países desenvolvidos, que podem ou não transferi-las para os países em desenvolvimento. Neste caso, os países em desenvolvimento necessitariam desenvolver tecnologias próprias para evitar maior dependência econômica em relação aos países desenvolvidos. A tecnologia intermédia iria se constituir num caminho alternativo para este grupo de países ainda com necessidades de desenvolvimento econômico.

\section{REFERÊNCIAS}

BARTON, J. R., "La Dimensión Norte-Sur de las Industrias de Limpieza Ambiental y la Difusión de Tecnologías Limpias”. Revista de la CEPAL, nº 64, p. 129-149, 1998.

CARSON, R., A Primavera Silenciosa. Lisboa: Pórtico, 1962.

CMMAD (Comissão Mundial sobre Meio Ambiente e Desenvolvimento), Nosso Futuro Comum. Rio de Janeiro: Fundação Getúlio Vargas, 1988

DASGUPTA, S.; LAPLANTE, B.; WANG, H. e WHEELER, D., "Confronting the Environmental Kuznets Curve", Journal of Economic Perspectives, vol. 16, $\mathrm{n}^{0} 1$, p. 147-168, 2002.

DOSI, G., "Institutions and Markets in a Dynamic World", The Manchester School, vol. LVI, no2, June, 1988.

FOXSON, T.; MAKUCH, Z.; MATA, M. e PEARSON, P., "Innovation Systems and Policy-Making Processes for the Transition to Sustainability". 
In: K. JACOB; M. BINDER e A. WIECZOREK (eds.), Governance for Industrial Transformation. Berlin: Environmental Policy Research Centre, p. 96-112, 2004.

GRETHER, J-M. e MELO, J. de, "Globalization and Dirty Industries: do pollution havens matter?”. NBER, Working Paper $\mathrm{n}^{\circ}$ 9776, 2003.

GROSSMAN, G. M. e KRUEGER, A. B., "Environmental Impacts of a North American Free Trade Agreement". NBER, Working Paper $n^{0}$ $3914,1991$.

HETTIGE, H.; MANI, M. e WHEELER, D., "Industrial Pollution in Economic Development: the environmental Kuznets curve revisited". Journal of Development Economics, vol. 62, p. 445-476, 2000.

ISLAM, S. M. N.; MUNASINGHE, M. e CLARKE, M., "Making LongTerm Economic Growth more Sustainable: evaluating the costs and benefits". Ecological Economics, vol. 47, p. 149-166, 2003.

JORGENSEN, D.W.; CHRISTENSEN, L.R. eLAU,L.J., "Transcendental Logarithmic Production Frontiers", The Review of Economics and Statistics, vol. 55, p. 28-45, 1973.

KUZNETS, S., "Economic Growth and Income Inequality", American Economic Review, vol. XLV, $n^{0} 1$, p. 1-28, 1955.

LEWIS, A., The Theory of Economic Growth. London: Allen and Unwin, 1955.

LEWIS, A., "Desenvolvimento Econômico com Oferta Ilimitada de Mãode-Obra". In: N. AGARWALA e S. P. SINGH (orgs.), A Economia do Subdesenvolvimento. Rio de Janeiro: Forense, p. 406-456, 1969.

LEWIS, A., "Development Economics in the 1950s". In: G. M. MEIER e D. SEEERS (eds.), Pioneers in Development. New York: Oxford University Press, p. 121-137, 1984.

LOKTA, A., "The Law of Evolution as a Maximal Principle", Human Biology, vol.17, p. 67-194, 1945.

MEADOWS, D. e MEADOWS, D., The Limits of Growth: a report 
for the Club of Rome's project on the predicament of Mankind. London: Potomac, 1972.

MOOMAW, W. R. e UNRUH, G. C., "Are Environmental Kuznets Curves Misleading Us?: the case of CO2 emissions". Environment and Development Economics, vol. 2 , $\mathrm{n}^{0}$ 4, p. 451-463, 1997.

MOTTA, R. S. da; FERRAZ, C. e YOUNG, C. E. F., Making Long-Term Growth More Sustainable: Brazil country case-study, final report for the project "Making Long-Term Growth More Sustainable" financed by the World Bank and coordinated by Mohan Munasinghe and Jorge Araújo, version October, mimeo., 2001.

MUNASINGHE, M., "Is Environmental Degradation an Inevitable Consequence of Economic Growth: tunneling through the environmental Kuznets curve". Ecological Economics, vol. 29, p. 89-109, 1999.

NOBRE, M. e AMAZONAS, M. de C. (orgs.), Desenvolvimento Sustentável: a institucionalização de um conceito. Brasília: IBAMA, 2002.

OPSCHOOR, H., "Economic Growth, the Environment and Welfare: are they compatible?”, Planejamento e Políticas Públicas, $n^{0}$ 18, p. 13-48, 1998.

PANAYOTOU, T., "Demystifying the Environmental Kuznets Curve: turning a black box into a policy tool', Environment and Development Economics, vol.2, $\mathrm{n}^{\mathrm{0}}$ 4, p. 465-484, 1997.

PANAYOTOU, T., "Economic Growth and the Environment", paper presented at the Spring Seminar of the United Nations Economic Commission for Europe, Geneva, March3, 2003.

RIBAUlT, J. M.; MARTINET, B. e LEBIDOIS, D., A Gestão das Tecnologias. Lisboa: Dom Quixote, 1995.

SACHS, I., Ecodesenvolvimento: crescer sem destruir. São Paulo: Vértice, 1986. 
SCHUMACHER, E. F., Good Work. New York: Harper and Rows, 1979 .

SCHUMACHER, E. F., O Negócio é Ser Pequeno: um estudo de economia que leva em conta as pessoas. Rio de Janeiro: Zahar, 1983.

SCHMIDHEINY, S., Changing Course: a global business perspective on development and the environment. Cambridge: The MIT Press, 1992.

SELDEN, T. M. e SONG, D., "Environmental Quality and Development: is there a Kuznets curve for air pollution emissions?", Journal of Environmental Economics and Management, vol. 27, p. 147$162,1994$.

SHAFIK, N. e BANDYOPADHYAY, S., Economic Growth and Environmental Quality: time series and cross country evidence, Washington. D. C.: The World Bank, 1992.

SOLOW, R. M., "The Economics of Resources or the Resources of Economics". American Economic Review, vol. 64,nº 2, p. 1-14, 1974.

SURI, V. e CHAPMAN, D., "The Economic Growth, Trade and Energy: implications for the environmental Kuznets curve", Ecological Economics, vol. 25, p. 195-208, 1998.

TIGRE, P. B., "Inovação e Teorias da Firma em Três Paradigmas", Revista de Economia Contemporânea, ${ }^{0}$ 3, p. 67-111, 1998.

TORRAS, M. e BOYCE, J. K., "Income Inequality and Pollution: a reassessment of the environmental Kuznets curve", Ecological economics, vol. 25, p. 147-60, 1998.

TURNER, R. K.; PEARCE, D. e BATEMAN, I., Environmental Economics: an elementary introduction. Baltimore: Johns Hopkins University Press, 1993.

VINCENT, J. R. "Testing for Environmental Kuznets Curves within a Developing Country", Environment and Development Economics, vol. 2 , no 4, p. 417-431, 1997.

WORLD BANK, The World Bank Development Report 1992: 
revista tecnologia e sociedade

development and the environment, New York: Oxford University Press, 1992.

WORLD BANK, World Development Indicators. Washington, D. C.: The World Bank, 2005. 